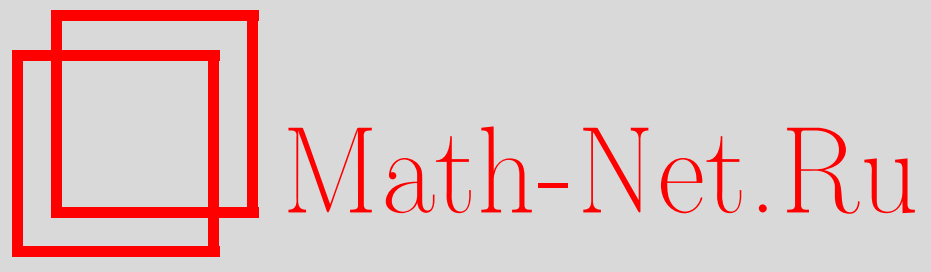

Н. Б. Мельников, Экстремальные свойства особенностей распределения Гурса, УМН, 2006, том 61, выпуск 4, 191192

DOI: https://doi.org/10.4213/rm1742

Использование Общероссийского математического портала Math-Net.Ru подразумевает, что вы прочитали и согласны с пользовательским соглашением http://www . mathnet.ru/rus/agreement

Параметры загрузки:

IP: 3.80 .181 .102

26 апреля 2023 г., 16:24:09

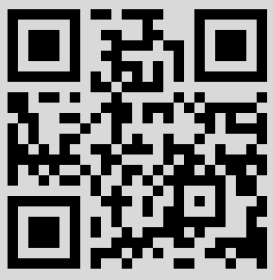




\section{Экстремальные свойства особенностей распределения Гурса}

\section{Н. Б. Мельников}

Распределение $D$ коранга $d \geqslant 2$ на многообразии $M$ размерности $m \geqslant 4$ называется распределением Гурса, если $D^{i+1}=D^{i}+\left[D^{i}, D^{i}\right], D^{1}=D$, является распределением при каждом $i=1, \ldots, d$ и выполнено условие $\operatorname{dim} D^{i+1}=\operatorname{dim} D^{i}+1$. Через $[X, Y], X, Y \in D$, обозначаем коммутатор векторных полей из $D$. Набор $\left(\operatorname{dim} D^{1}(q), \operatorname{dim} D^{2}(q), \ldots\right)$ называется большим вектором роста распределения $D$ (не обязательно Гурса) в точке $q \in M$. Малым вектором роста распределения $D$ в точке $q \in M$ называется набор $\left(\operatorname{dim} D_{1}(q), \operatorname{dim} D_{2}(q), \ldots\right)$, где $D_{i+1}=D_{i}+\left[D, D_{i}\right]\left(D_{1}=D\right)$. $\mathrm{B}$ точке общего положения распределения Гурса большой и малый векторы роста совпадают и равны $(r, r+1, r+2, \ldots, m), r=m-d$. Точки, в которых это условие не выполнено, называются особыми.

Распределение $\operatorname{Span}\left(f_{0}, f_{1}\right)$, порожденное парой векторных полей

$$
f_{0}(q)=\left(v_{0} \cos \theta_{0}, v_{0} \sin \theta_{0}, v_{1} \sin \left(\theta_{1}-\theta_{0}\right), \ldots, v_{i} \sin \left(\theta_{i}-\theta_{i-1}\right), \ldots, v_{n} \sin \left(\theta_{n}-\theta_{n-1}\right), 0\right),
$$

$$
v_{i}=\prod_{j=i+1}^{n} \cos \left(\theta_{j}-\theta_{j-1}\right), \quad i=0,1, \ldots,(n-1), v_{n}=1, \quad f_{1}(q)=(0, \ldots, 0,1),
$$

является распределением Гурса на $\mathbb{R}^{2} \times\left(S^{1}\right)^{n+1}$ с координатами $q=\left(x, y, \theta_{0}, \ldots, \theta_{n}\right)$ (см. [1]) и моделирует все возможные особенности произвольного распределения Гурса коранга $n+1[2]$.

Особенности распределения $\operatorname{Span}\left(f_{0}, f_{1}\right)$ задаются одним из соотношений: $\cos \left(\theta_{k}-\theta_{k-1}\right)=0, \quad k=2, \ldots, n$ (см. [1]). М. И. Зеликиным (частное сообщение, 2001) была высказана гипотеза о том, что такие кривые могут быть описаны как особые оптимальные траектории задачи $T \longrightarrow \min , \dot{q}=f_{0}(q)+u f_{1}(q), q(0)=a$, $q(T)=b,|u| \leqslant \omega$. В работе [3] была доказана оптимальность (сильный минимум) малых участков одной из $n-1$ таких особенностей. В настоящей работе доказано, что достаточно малый участок любой особой траектории первого порядка соответствующей задачи доставляет слабый минимум (теорема 1). Более того, произвольная особенность указанного 2-распределения Гурса является особой траекторией, и любой ее участок - не обязательно малый - доставляет слабый минимум (теорема 2).

Исходную задачу быстродействия преобразуем к задаче на фиксированном отрезке введением дополнительной переменной:

$$
\mathscr{J}=z(0) \longrightarrow \min , \quad \dot{z}=0, \quad \dot{q}=z\left(f_{0}(q)+u f_{1}(q)\right), \quad t \in\left[0, t_{1}\right] .
$$

Исследуем получившуюся задачу на минимум среди всех абсолютно непрерывных вектор-функций $q(t)$ и измеримых скалярных функций $u(t)$. Слабый минимум в задаче (3) задается нормой $|z|+\|x\|_{C}+\|u\|_{\infty}$; сильный минимум задается полунормой $|z|+\|x\|_{C}$.

Обозначим траекторию задачи (3) через $\widehat{w}(t)=(\widehat{z}(t), \widehat{q}(t), \widehat{u}(t)), t \in\left[0, t_{1}\right]$ (не ограничивая общности, считаем $\widehat{z}=1, t_{1}=\widehat{T}$ ). Набором множителей Лагранжа назовем пару $\lambda=\left(\psi, \alpha_{0}\right)$, где липшицева функция $\psi(t)$ - решение сопряженной системы $\dot{\psi}=-\psi \widehat{f}_{0}^{\prime}$ и число $\alpha_{0} \geqslant 0\left(\|\psi\|_{C}+\alpha_{0} \neq 0\right)$ таковы, что выполняется условие максимума: $\max _{|u| \leqslant \omega} H[\lambda](\widehat{z}, \widehat{q}, u)=H[\lambda](\widehat{z}, \widehat{q}, \widehat{u})=\psi\left(f_{0}(\widehat{q})+\widehat{u} f_{1}(\widehat{q})\right)=\alpha_{0} / \widehat{T} \geqslant 0$.

Траектория $\widehat{w}$ задачи (3) называется особой, если управление не входит в условие максимума: $\psi \widehat{f_{1}}=0$. Порядок особой траектории $\widehat{w}$ равен единице, если $\psi\left[\widehat{f_{0}, f_{1}}\right]=0$ и $\left.\psi\left[f_{1}, \widehat{\left[f_{0}, f_{1}\right.}\right]\right] \neq 0$.

Траектория называется регулярной (нормальной), если существует такой набор множителей Лагранжа $\lambda=\left(\psi, \alpha_{0}\right)$, что $\alpha_{0} \neq 0$. Преобразование Гоха (см. [4]) на 
регулярной особой траектории задачи (1)-(3) может быть записано в виде $(\bar{q}, \bar{u}) \mapsto$ $(\bar{\xi}, \bar{v})$, где $\bar{\xi}=\bar{q}-\bar{v} \widehat{f}_{1}, \dot{\bar{v}}=\bar{u}, \bar{v}(0)=0$.

Лемма 1. Пусть $\widehat{w}$ - регулярная особая траектория. Тогда в переменных Гоха критические вариации задаются системой $\dot{\bar{\xi}}=\widehat{f_{0}^{\prime}} \bar{\xi}-\bar{v}\left[\widehat{f_{0}, f_{1}}\right], \bar{\xi}(0)=0$, и вторая вариация имеет вид

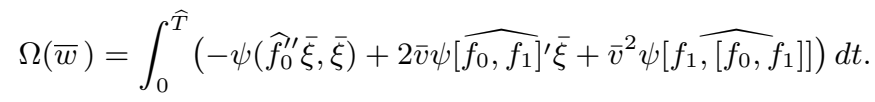

Лемма 2. Пусть $f_{0}, f_{1}$ заданы формулами (1), (2). Тогда $\left[f_{1},\left[f_{0}, f_{1}\right]\right]=f_{0}$.

Теорема 1. Малье участки любой особой траектории первого порядка доставляют слабый минимум в задаче (1)-(3).

СХемА доКАЗАТЕЛьствА. Из леммы 2 и условия максимума следует, что на особой траектории первого порядка выполняется строгое условие Келли: $\psi\left[f_{1},\left[f_{0}, f_{1}\right]\right]>0$. Это условие обеспечивает положительную определенность квадратичной формы (4) на малых отрезках времени (см. [4]).

Теорема 2. Пусть $\gamma$-допустимая траектория задачи (1)-(3), принадлежащая поверхности $\Gamma_{k}=\left\{\cos \left(\theta_{k}-\theta_{k-1}\right)=0\right\}, k=1, \ldots, n, u \omega>1$. Тогда $\gamma$ является особой траекторией первого порядка и доставляет слабый минимум в задаче (1)-(3).

СХема ДоказАтЕЛЬСТвА. Непосредственно проверяется, что на кривой $\gamma \subset \Gamma_{k}$ управление $\widehat{u}= \pm(n-k+1)^{-1 / 2}$ внутреннее: $|\widehat{u}|<\omega$, следовательно, $\gamma \subset \Gamma_{k}-$ особая траектория. Кроме того, $\psi(t)=(0, \ldots, 0,1, \ldots, 1,0)$, где единицы начинаются с $(k+2)$-й координаты, - решение сопряженной системы. Тогда вторая вариация имеет вид

$$
\Omega(\bar{w})=(n-k+1)^{-1 / 2} \int_{0}^{\widehat{T}} \sum_{j=k+3}^{n+3}(j-k-2)\left(\bar{q}_{j}-\bar{q}_{j-1}\right)^{2} d t
$$

и является положительно определенной квадратичной формой при любом $\widehat{T}$.

ЗАмечАниЕ. В случае $\omega=1$ кривая $\gamma \subset \Gamma_{n}$ доставляет сильный минимум.

Работа выполнена при финансовой поддержке Фонда содействия отечественной науке. Автор благодарен М. И. Зеликину и А. В. Дмитруку за полезные обсуждения.

\section{Список литературы}

[1] F. Jean, ESAIM Control Optim. Calc. Var., 1 (1996), 241-266. [2] R. Montgomery, M. Zhitomirskii, Ann. Inst. H. Poincaré Anal. Non Linéaire, 18:4 (2001), 459-493. [3] О.Е.Долгалева, Вестн. МГУ. Сер. 1. Матем., мех., 2005, № 5, 34-39. [4] A. V. Dmitruk, Proc. Sympos. Pure Math., 64 (1999), 163-198.

Н. Б. Мельников (N. В. Melnikov)

Центральный экономико-математический институт РАН; Московский государственный университет им. М. В. Ломоносова

E-mail: melnikov@cs.msu.su
Представлено М. И. Зеликиным Принято редколлегией 21.03.2006 